\title{
Wirelessroboticarm Vehicle
}

\author{
P. V. Rama Raju, G. Naga Raju, K. Venkat Vinay, M. Anil Kumar, K. Naga Manju, K. Manoj
}

\begin{abstract}
Today, advance is making a relative course according to quickly extending human needs. The work done to address these issues makes life less magnificent continually, and these examinations are moved in electronic arm considers. Robot arms work with an outside client or by performing foreordained headings. These days, the most made field of robot arms in each field is the business and medication area. Managed and found in the endeavor, the robot arm can move in 4 turn ways with 5 servo engines. Because of the holder, you can take the ideal material start with one spot and pass on it then onto the going with spot, and moreover blend it with the material it gets. While doing this, robot control is given by work together with the android application by methods for Bluetooth module related with Arduino Uno microcontroller.The basic inspiration driving social occasion of the undertaking was to structure and build up the bit of mechanical arm for lifting. The motorized arm was composed with four degrees of shot and programming to achieve totally direct light material lifting errand to engage the age to lie in any industry. 3d printing framework is utilized in this test to creation. The zones of the mechanical arm is furnished with 5 servo engines to interface the parts and bringing arm improvement. Arduino, an open source control mechanical assembly and making PC exercises is associated with control the mechanized arm by driving servo engines to be set up to change the positions. Remote control was finished by utilizing a PDA with android working structure through a Bluetooth module. The mechanical arm was under endeavoring and supporting its presentation and the outcomes shows that it can play out the lifting task fittingly.
\end{abstract}

Keywords-Flex sensors; MIT app; Arduino; Servo Motors; Robotic arm; Node MCU

\section{INTRODUCTION:}

Nowadays' family dependably needed extra support structures. With the lively augmentation in the improvement of data, individuals are really guided to scan for changed markets and individuals have entered the test to make quality things monetarily. Mechanization frameworks are furthermore expected to get this. Since composed robotization frameworks are required to tie disordered stacks up generally as to have encountered and strong and devoted workers for quality things. In light of their physical properties, individuals expected to utilize partner machines in spots where their quality was deficient. These machines, which are worked with the prerequisite for human help with headway, have been made to work solidly without the need of human power with the advancement of advancement. A legend among the most utilized portions of robotization

Revised Manuscript Received on April 12, 2019.

Dr. P. V. Rama Raju,Professor \& HOD,Department of ECE, SRKR Engineering College (A), Bhimavaram, A.P, India..

G. Naga Raju,Professor,Department of ECE, SRKR Engineering College (A), Bhimavaram, A.P, India..

K. Venkat Vinay,B.E Students, Department of ECE, SRKR Engineering College (A), Bhimavaram, A.P, India.

M. Anil Kumar,B.E Students, Department of ECE, SRKR Engineering College (A), Bhimavaram, A.P, India..

K. Naga Manju,B.E Students, Department of ECE, SRKR Engineering College (A), Bhimavaram, A.P, India..

K. Manoj, B.E Students, Department of ECE, SRKR Engineering College (A), Bhimavaram, A.P, India. structures is robots. Robotized structures; Mechanical Engineering, Electrical Engineering and Computer Engineering have all gotten together to share. In the undertaking, specialists have been done and perceived to consider mechanics and programming during the activities wrapped up by the robot arm which is proposed to satisfy the assignments picked by foreordained headings. In any case, it was settled what most remote point the robot arm would be and what upgrades it could make. Computerized arm made of Android telephone or tablet control; it can pass on the ideal material, blend it up and play out the headings starting late obliged by a client. In the event that this undertaking is in like way a doled out errand; the robotized arm takes a bit of material and passes on it to the ideal position and a short timeframe later records its upgrades and engages it to finish a similar headway until we stop it. The servomotor is kept up so as to have the choice to play out these assignments reasonably since the engine to be picked must work no doubt and should be at high torque. The robot arm is made out of 5 servo engines and can move in 4 focus point ways with these engines. In the undertaking, Arduino Uno microcontroller is changed and servo engine control is given. As such, it is conceivable to play out the ideal activities by structures for the parts dealt with on the Arduino with no circuit advance other than the circuit where the servo engine information sources are found. For the mechanical part, the robot arm is drawn with the Solid Works program and the areas of the robot arm are outlined.

The remainder of the paper is made as scans for after. Section 2 delineates the survey of past papers. Section 3 and 4 depicts apparatus and programming instruments utilized in our structure solely. Zone 4 depicts the structure plan and stream design. Section5 follows foundation results and section6 portrays the end and future improvement.

\section{RELATED WORK:}

There are different tendencies by which a robotized arm might be controlled. In the past there have been different specialists endeavoring to control motorized arm through work stations, Joysticks, in spite of interfacing them with the web so they can be controlled from any place on the planet. Usually the vast majority of the mechanical arms are obliged by a focal controller which makes occupations of Human Arm Movement Sensors (Accelerometers) Atmega32 Microcontroller Processing Unit Atmega640 Microcontroller Robotic Arm Actuators (Servo Motor Controller) 4 respects taken in from the terminal that are entered by the client at the terminal to move the arm to a specific personalities in space. This makes the control incredibly hazardous as the control estimations of the 
engines are remarkably hard to anticipate to accomplish a specific movement.And beginning late, a bleeding edge robot with six joints vivaciously takes after a human arm - it has what might be showed up unmistakably in association with a shoulder, an elbow and a wrist. Commonly, the shoulder is mounted to a stationary base structure rather than a lessened body. This kind of robot has six degrees of shot, which means it can turn in six remarkable ways. A human arm, by relationship, has seven degrees of freedom. The arm's essential responsibility is to move your hand everything considered. Essentially, the robotized arm's huge responsibility is to move an end effector everything considered. You can outfit modernized arms with a wide level of end effectors, which are fit to a specific application. One standard end effector is a disentangled form of the hand, which can regulate and pass on various articles. Robotized hands dependably have worked in weight sensors that tell the PC how hard the robot is understanding a specific article. This shields the robot from dropping or breaking whatever, it's passing on. Far edge effectors join blowtorches, bores and shower painters.Industrial robots are relied upon to accomplish something by and large identical to, in a controlled condition, again and again. For instance, a robot may wind the tops onto nutty spread compartments sliding a mechanical age structure. To incite a robot the most ideal approach to manage consummation its responsibility, the program manages the arm through the updates utilizing a handheld controller. The robot stores the careful advancement of upgrades in its memory, and does it over and over each time another unit hops the gathering line.Most current robots work in auto sequential movement structures, gathering vehicles. Robots can complete a gigantic extent of this work more satisfactorily than people since they are so careful. They generally drill in a close spot, and they as a rule fix stumbles with an essentially unclear level of force, paying little notice to what degree they've been working. Amassing robots are besides central in the PC business. It takes a strikingly careful hand to aggregate a little microchip.

\section{HARDWARE COMPONENTS:}

- Arduino Uno little scale controller

- Servo engines

- Node MCU little scale controller

- Nrf24L01

- Modules

- Flex sensors

- DC engines

- Motor driver

- Power Antenna

\section{PROGRAMMING TOOLS:}

- Arduino IDE

- MIT application Inventor

\section{SYSTEM ARCHITECTURE:}

In the transmitter end, with the intercession of the flex sensors that are related with the ArduinoUno microcontroller, we can control the mechanical arm which was fixed at the beneficiary end. The developments of this territory were completely picked the flex sensors those are fixed on a glove at the transmitter side, in this manner the robotized arm was performed subject to our ideal activities.



Fig 1: Transmitter End

Nrf24L01 is the handset that for the most part used to change over the key sign to robotized sign to the servos those are utilized to move the fingers of the electronic arm



Fig 2:Transceiver Block Diagram

Flex sensors are mounted on each joint of the greater part of the five fingers and turning of sensor in perspective close by advancement of the principle changes the constrainment of the sensor and this adjustment in hindrance is asked as certification to the mechanical unit.



Fig 3: Flex Sensors

Following fig indicates exploratory framework which contains transmitter for example glove mounted on human palm with flex sensors and ace which is robot controller arm. This arrangement demonstrates recipient is following improvement of transmitter.

Various structures can be utilized for seeing attestation of the thing. For the key keep running of the errand the covering based affirmation has been picked. Since the optical sensors are utilized missing much by technique for treatment of the information 
the seeing is done at a snappier rate. The hiding sensor itself offer direct snippet of data to the microcontroller jumping out at seeing the article. The basic errand of the microcontroller is limited to servo engine control and the general reaction is relied upon to be better. The L293D driver ICs are utilized to drive the DC servo engines. The drivers are enthusiastic enough to pass on the beats at anticipated that speed should the engines. This undertaking is a blend of four to five more humble than run of the mill works out. This is, in our playing out various errands robot we have remote mechanical hand, it may be constrained by a glove that we wear and moreover these control from the glove are move to the beneficiary zone that is, our model robotized hand through nrf24101 handset. Thusly, the relating sign acts concurring and the yield is showed up through the turn of seeing servo engines. We are going to structure a device that has both mechanical arm and modernized hand as the single undertaking that we can expect the occupations of our project.In our endeavor we have robotized hand related with an electronic arm, these as we may acknowledge from this set up, we have steadfast human hand and we can perform most conspicuous potential activities that a human hand does. To made this whole set up mobiles all over this surface(movable) we are going to configuration surface base to this methodology. To contraption this method remote we utilize focus point mcu progressively little scale controller bordering the servo engines and thus in our undertaking $\mathrm{t}$ we are demonstrating wi-fi improvement.

We can control this undertaking up to a customary degree of 100 meters from our technique. To utilize this whole methodology in affirmations, for instance in bomb ID applications, we are going to place land mine marker at the front fulfillment of the base with the target that it will give some go to the locator. Absolutely when the bomb is recalled that we can wreck it by utilizing our mechanical hand. In extra to this, we can have recognition camera as the front of our base and it acts a perception robot. To us this robot in endeavors like picking and squashing articles we utilize a covering identifier circuit on the palm of the automated hand. This, the yield from the automated hand picks the headway that will be performed future.

\section{Fig 4:Real time existing model}

\subsection{FLOW CHART:}
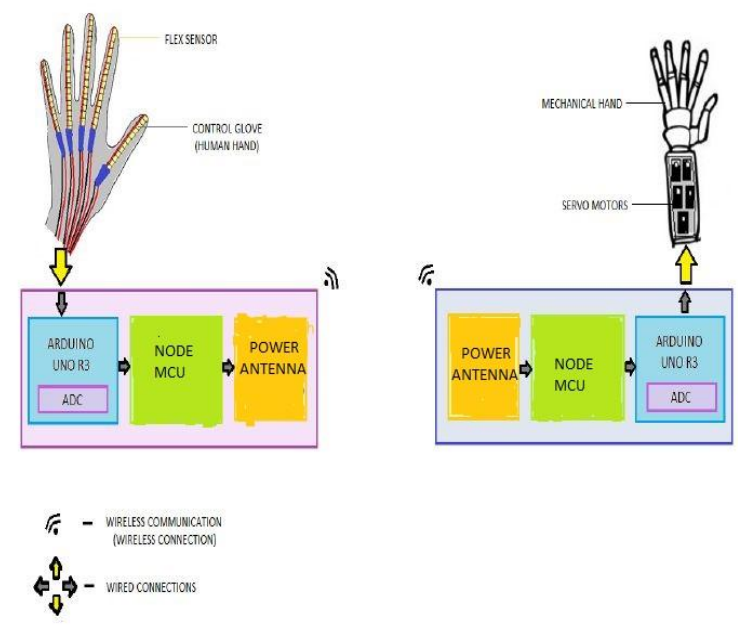

Fig 5: Flow Chart for Robotic Hand

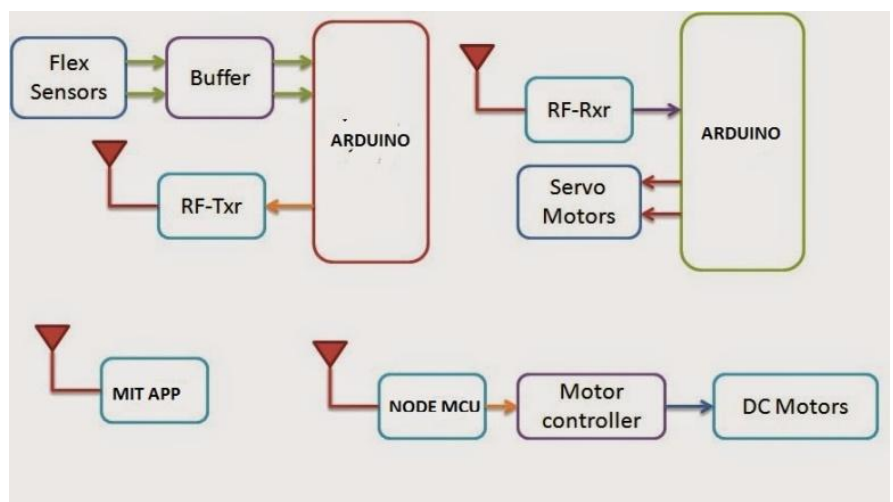

Fig 6: Flow Chart for Robotic vehicle

\section{RESULTS:}

Flex Sensor Output Variation: Figure 7 demonstrates the social event of volt concerning collection in avoiding of flex sensor. At first the flex sensor is at default position, a solid estimation of 73 degree. The worth reductions as we begin bowing the flex sensor. This specific change in flex sensor worth is utilized to turn servo engines.

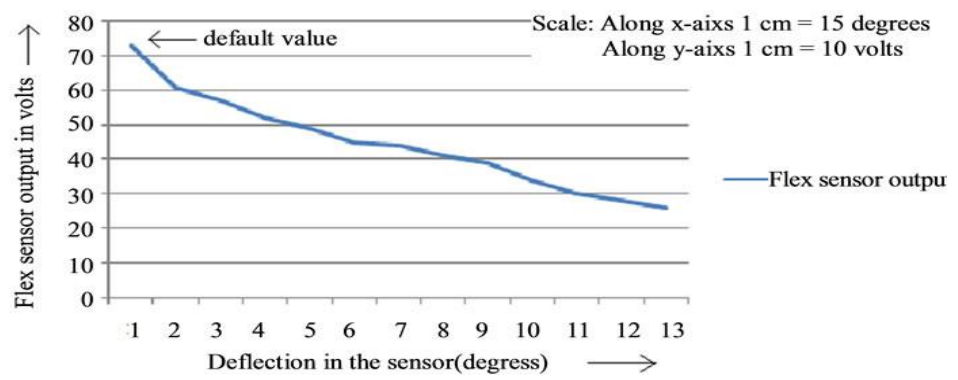

Fig 7: Deflection in the sensor V/S flex sensor output.

Servo Motor Variation: Fig 8 shows the servo engine assembling as showed up by the PWM signal which picks the turn of the servo engine in various edges. 




Fig 8: Servo Motor PWM Timing Diagram

\section{CONCLUSION AND FUTURE SCOPE:}

Mechanical arms, different regions are developable. In light of the mechanical arms, different assignments are made progressively prompt and the resulting destruction up level has been reduced to a base. For instance; some remedy store based fix giving robots and an anticipated robot arm have been made. Likewise, the capacity to move the robot arm is moreover broadened, and when the camera is set in the finger a territory and the affectability is extended, it will everything considered be utilized in a wide degree of occupations from the therapeutic division to the robotization structures. With the robotized arms made in like way, the danger of sullying the patient in the pleasing zone is confined, while the human amazes are obliged during the cautious mediation. Despite the way wherein that the motorized arm made by this undertaking is of model quality, it has a quality that can be improved for dependably mechanical structures. Other than these, mechanical arm division, which is available to advancement, will keep its monstrosity later on. The motivation driving the errand is to give control of 4 tomahawks moving robot arm structure and this robot arm with a reasonable microcontroller and Bluetooth module with android application. The fundamental speculative and reasonable data along these lines has been gotten and the crucial foundation has been made for the undertaking. During the course toward making and stirring up the task, a great deal of hypothetical learning has been moved to the arrangement and it has been guaranteed that it is reasonable with the veritable focal point of the undertaking.

\section{REFERENCES:}

1. Mohd Ashiq Kamaril Yusoffa, Reza Ezuan Saminb, Babul Salam Kader Ibrahimc, "WirelessMobile Robotic Arm", International Symposium on Robotics and Intelligent Sensors 2012 (IRIS2012), July 2012

2. Wan Muhamad Hanif Wan Kadir, Reza Ezuan Samin, Babul Salam Kader Ibrahim, "InternetController Robotic Arm". International Symposium on Robotics and Intelligent Sensors 2012 (IRIS2012), July 2012

3. Avinash Jain, "Servo Motor Control by Using AVR ATmega32

Microcontroller",http://extremeelectronics.co.in/avrtutorials/servo-motor-control-by-using-avr-atmega32microcontroller/, June 2010

4. Arvind Kumar Saini1, Garima Sharma2, Kamal Kishor Choure3, "BluBO: Bluetooth ControlledRobot," International Journal of Science and Research (IJSR) National Conference on Knowledge,Innovation in Technology and Engineering (NCKITE), 10-11 April 2015, pp. 325-328S.

5. Arpit Sharma1, Reetesh Varma2, Saurabh Gupta3 and Sukhdeep Bhatia4, "Android PhoneControlled Robot Using Bluetooth" IJEEE ISSN 0974-2174, Volume 7, Number 5 (2014, pp. 443-448)

6. M.Selvam1, "Smart phone based robotic smart phone based robotic" IJRET Volume: 03 Issue:03, Mar-2014, pp. 229-232.



\section{ABOUT AUTHORS:}

\section{Dr.P.V.Rama Raju}

Presently working as a Professor and HOD of Department of Electronics and Communication Engineering, S.R.K.R. Engineering College, AP, India. His research interests include BiomedicalSignal Processing, Signal Processing, Image Processing, VLSI Design, Antennas and Microwave Anechoic Chambers Design. He is author of several research studies published in national and international journals and conferenceproceedings.

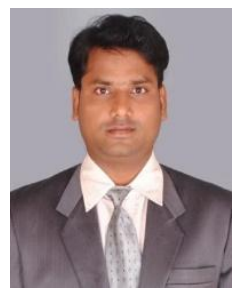

\section{G. Naga Raju}

Presently working as assistant professor in Dept. of ECE, S.R.K.R. Engineering College, Bhimavaram, AP, India. He received B.Tech degree from S.R.K.R Engineering College, Bhimavaram in 2002, and M.Tech degree in Computer electronics specialization from Govt. College of Engineering., Pune University in 2004. His current research interests include Image processing, digital security systems, Signal processing, Biomedical Signalprocessing, and VLSI Design.

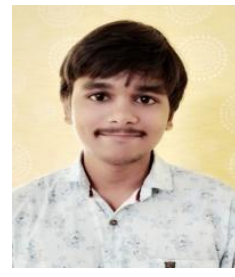

\section{K. Venkat Vinay}

Presently pursuing Bachelor of Engineering degree in Electronics \& Communication engineering at S.R.K.R. Engineering College, AP, India.

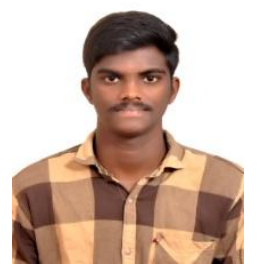

\section{Anil Kumar}

Presently pursuing Bachelor of Engineering degree in Electronics \& Communicationengineering at S.R.K.R. Engineering College, AP, India.

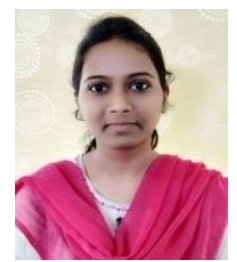

\section{K. Naga Manju}

Presently pursuing Bachelor of Engineering degree in Electronics \& Communication engineering at S.R.K.R. Engineering College, AP, India.

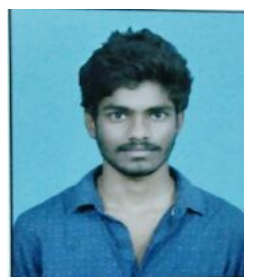

\section{K.Manoj}

Presently pursuing Bachelor of Engineering degree in Electronics \& Communication engineering at S.R.K.R. Engineering College, AP, India. 